Supporting Information for:

\title{
Crossover from Superexchange to Hopping as the Mechanism for Photoinduced Charge Transfer in DNA Conjugates
}

Frederick D. Lewis,${ }^{\dagger}{ }^{\dagger}$ Huihe Zhu ${ }^{\dagger}$ Pierre Daublain, ${ }^{\dagger}$ Torsten Fiebig, ${ }^{\ddagger}{ }^{\ddagger}$ Milen Raytchev,${ }^{\ddagger}$ Qiang Wang $^{\ddagger}$ and Vladimir Shafirovich ${ }^{\star \mathcal{8}}$

Departments of Chemistry, Northwestern University, Evanston, IL 60201, Boston College, Chestnut Hill, MA 02467, and New York University, New York, NY 10003

\section{Contents}

Figure S1. Transient fluorescence decays for 3 with 2 exp and 3 exponential fits.

Figure S2. Temporal evolution of the pump-probe spectra of (a) Sa and (b) Sd in the time range of 0.2 ns to 1.2 ns after excitation at 333nm.

Figure S3. Temporal evolution of the pump-probe spectra of 2, 3 and $\mathbf{4}$ in the time range of $0.2 \mathrm{~ns}$ to $1.9 \mathrm{~ns}$ after excitation at $355 \mathrm{~nm}, 333 \mathrm{~nm}$ and $333 \mathrm{~nm}$ respectively. 
Figure S1. Transient fluorescence decays for 3 with 2 exp and 3 exponential fits.
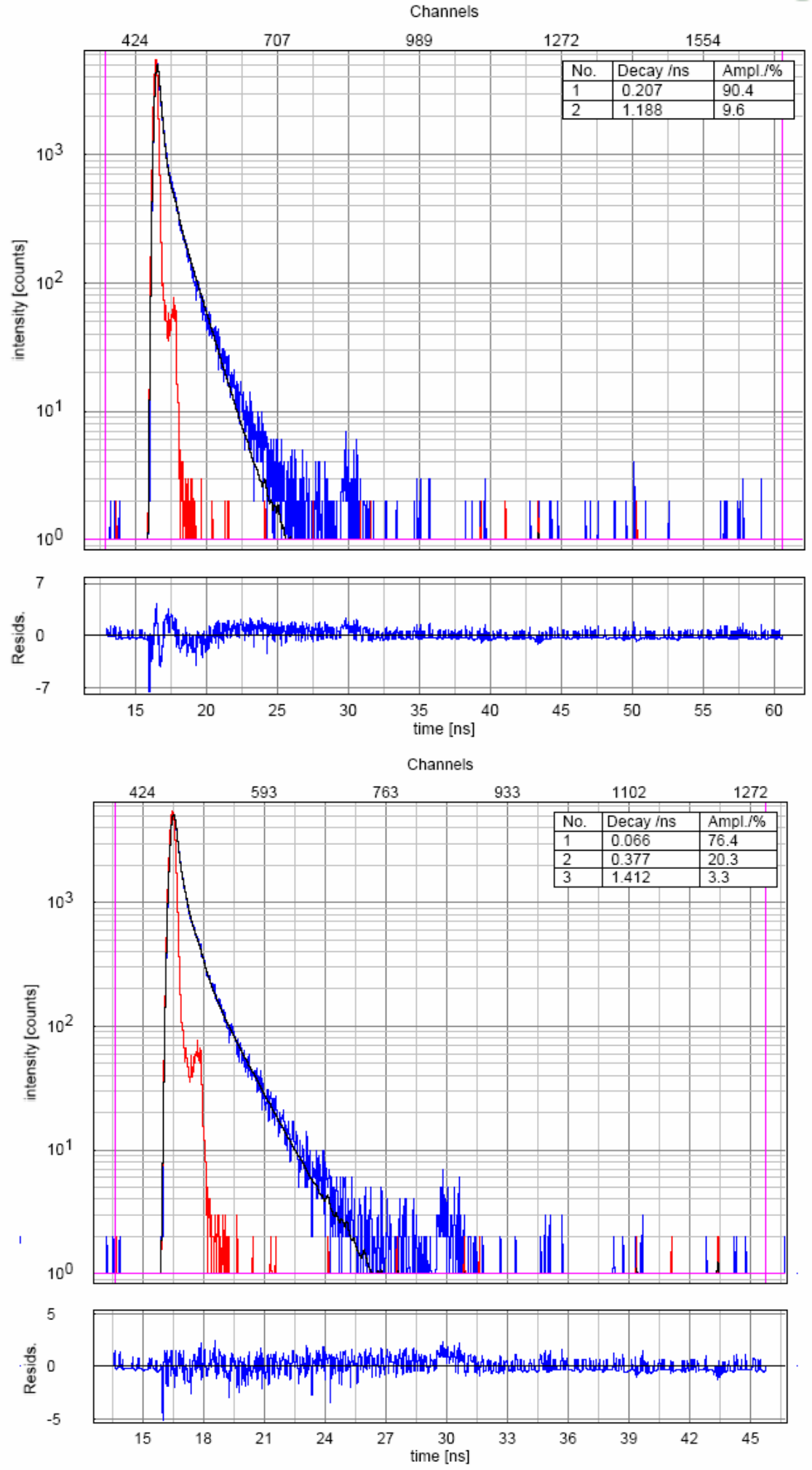
Figure S2. Temporal evolution of the pump-probe spectra of (a) Sa and (b) Sd in the time range of 0.2 ns to 1.2 ns after excitation at 333nm. Early spectra are shown in blue/green and late spectra are shown in orange/red colors. Decay times for Sa at $570 \mathrm{~nm}$ are: $1.8 \mathrm{ps}(-7.4)$ and $362 \mathrm{ps}$ (134.7). Decay times for Sd at $575 \mathrm{~nm}$ are: 2.8 ps (19\%), $7.6 \mathrm{ps}(-23 \%)$, and 379 ps (58\%).

(a)

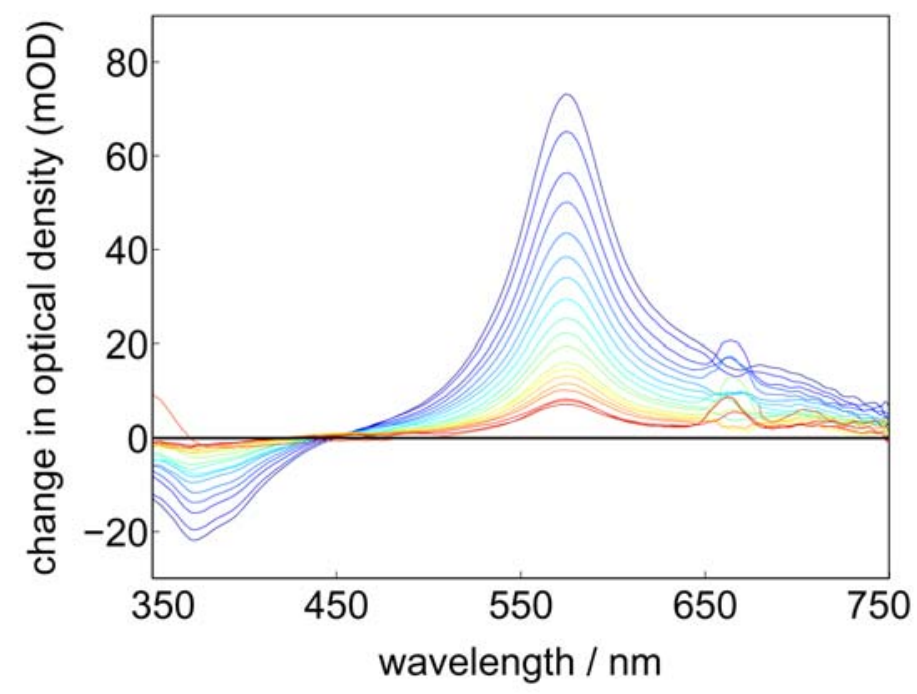

(b)

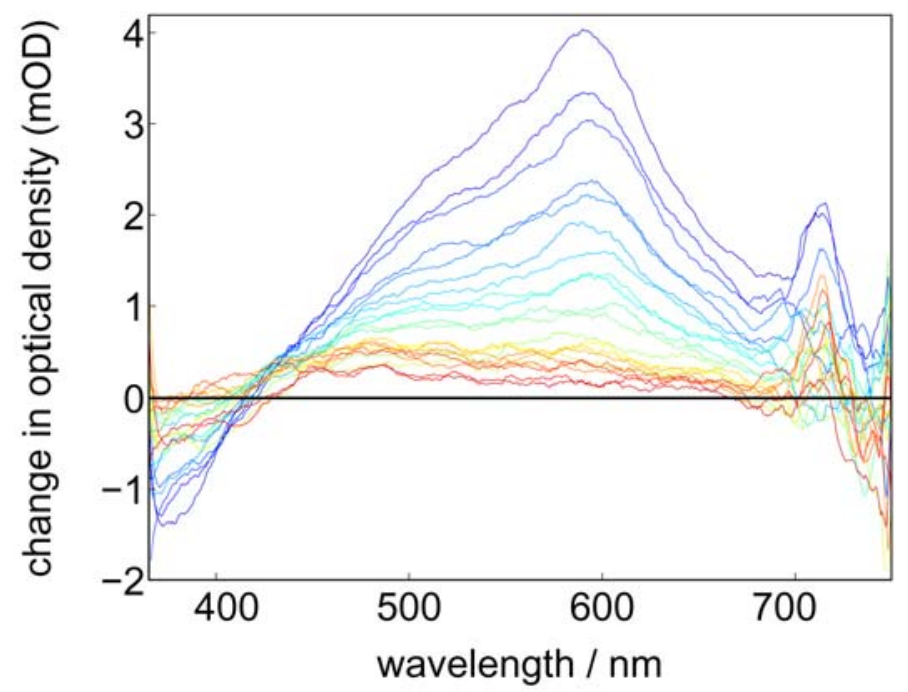


Figure S3. Temporal evolution of the pump-probe spectra of 2, $\mathbf{3}$ and $\mathbf{4}$ in the time range of $0.2 \mathrm{~ns}$ to $1.9 \mathrm{~ns}$ after excitation at $355 \mathrm{~nm}, 333 \mathrm{~nm}$ and $333 \mathrm{~nm}$ respectively. Early spectra are shown in blue/green and late spectra are shown in orange/red colors.

2

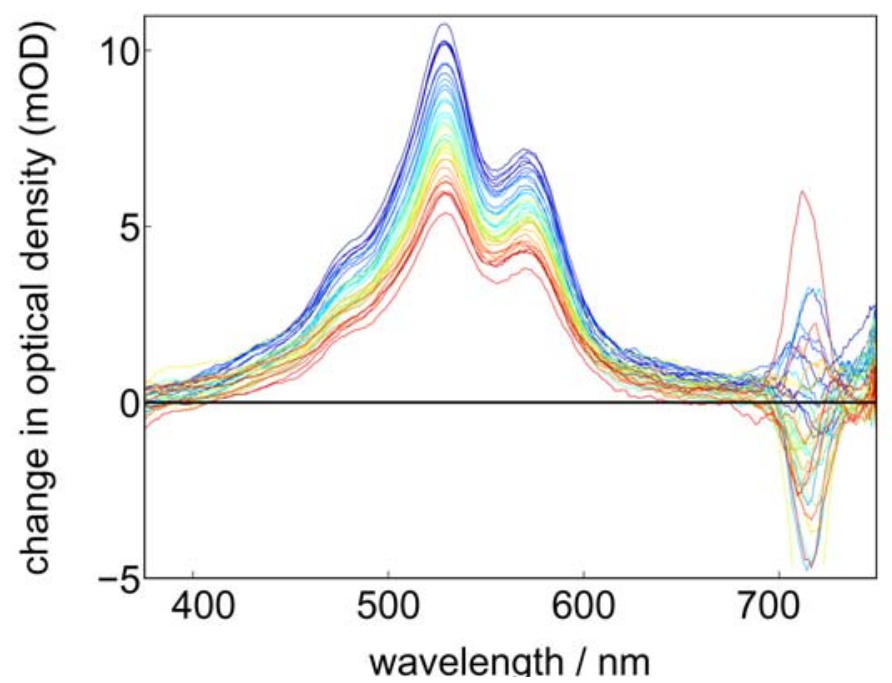

3

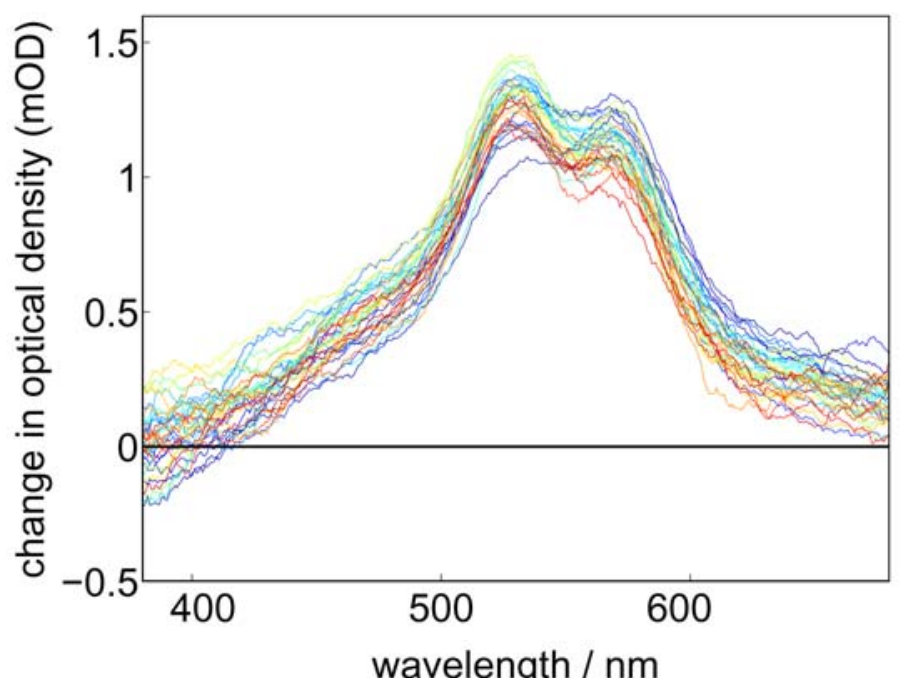

4

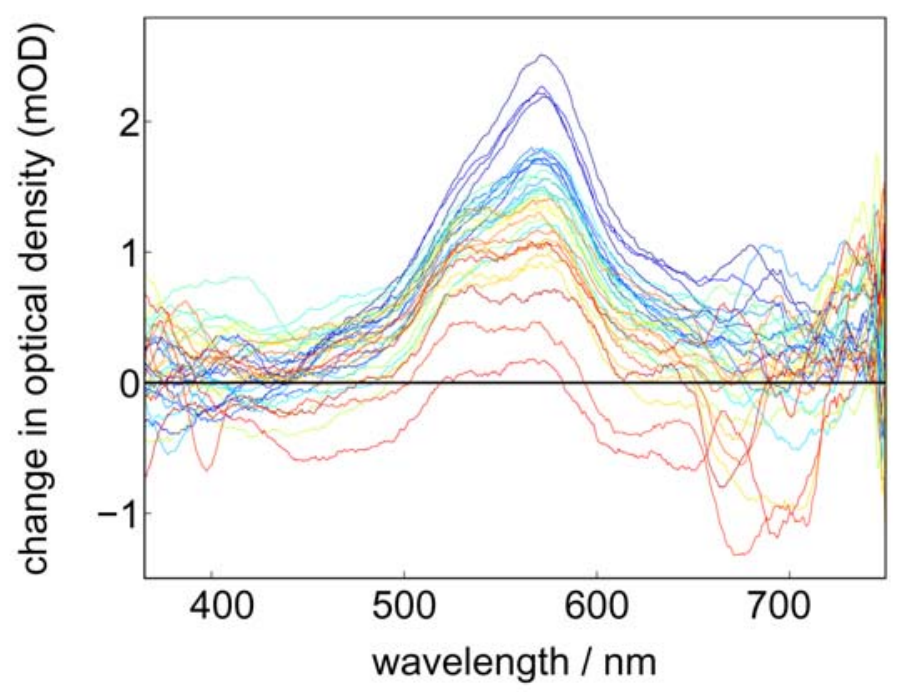

